\title{
DEVELOPMENT OF THE UNIVERSAL TOOL FOR TESTING OF TENSILE PROPERTIES OF HEXAGONAL STEEL WIRE MESH FOR CIVIL ENGINEERING
}

\author{
Ivana Atanasovska ${ }^{1 *}$, Dejan Momčilović ${ }^{2}$, Milorad Gavrilovski ${ }^{3}$ \\ ${ }^{I}$ Mathematical Institute of the Serbian Academy of Sciences and Arts, \\ Belgrade, Serbia \\ ${ }^{2}$ Institute for Testing of Materials, Belgrade, Serbia \\ ${ }^{3}$ Innovation Center of Faculty of Technology and Metallurgy, University of \\ Belgrade, Serbia
}

Received 09.06.2018

Accepted 22.06.2018

\begin{abstract}
The developing of the universal tool for testing of tensile properties of hexagonal steel wire mesh for civil engineering is described in this paper. The developed tool allows repeated testing of hexagonal steel wire mesh of different dimensions without tool changes and is generally related with the procedure for the determination of tensile strength properties of different wire meshes. The construction of the tool which is related to the aims of the decreased mass consumption and high operation safety is described in detail. Particular attention is focused on the safety component of the tool which ensuring safety testing by preventing slipping of the wire mesh samples during loading. The paper also presents the Finite Element Analysis performed in order to verify the high safety factor of the developed tool. The contact regions with stress concentration behavior are analyzed by non-linear solvers. The obtained results and conclusion about the possible contributions of the developed universal tool for extensively testing of wire meshes for civil engineering are discussed.
\end{abstract}

Keywords: tensile strength; wire mesh; Finite Element Analysis.

\section{Introduction}

Tensile testing machines are the essential equipment for testing mechanical properties of hexagonal steel wire mesh as a part of the regular quality control. However, the available and existing grips are commonly constructed for testing wires and various types of tensile specimens which means that they are not suitable for testing

\footnotetext{
*Corresponding author: Ivana Atanaskovska, iatanasovska@mi.sanu.ac.rs
} 
wire mesh. The primary goal of tensile testing machines adaptation is to make possible testing of various dimensions of wire meshes on one testing machine which requires costly adjustment of the gripping system. An alternative approach is to design and use specific tool with a gripping system that is compatible with existing one on the tensile testing machine. New design underlines the specific requirements of a tool for tensile testing of hexagonal steel wire mesh only, which are: user-friendly construction, availability for testing on many tensile machines and flexibility for all dimensions defined by existing standards. The rough sketch of such tools for testing hexagonal steel wire mesh in civil engineering applications is defined by standard EN 10223-3:2013 [1]. Available papers show that the main topic regarding the use of wire mesh for civil engineering describes the analytical and numerical approach as a simulation of testing under various conditions [2, 3]. In one of the available papers [4], authors show matching of testing results with a numerical model of wire mesh. The authors [4] constructed specific testing and gripping tool for wire mesh, for one dimension only and without applied safety measures. Similar tools with limited applicability, which means that the tool is suitable for testing only one dimension of wire mesh, can be found in production lines of some producers of testing equipment $[5,6]$.

\section{Description of the developed tool}

The universal tool for testing of tensile properties of hexagonal steel wire mesh for civil engineering is developed to allow extensive and repeated testing of hexagonal steel wire mesh of different dimensions without tool changes and filed for R.S. Patent [7]. The basic construction of the developed universal tool is shown on Fig.1 and Fig.2 by one of two identical pieces of the tool with main parts. The testing by the tool is carrying out by positioning one by one of two identical pieces of the tool by the holder (5) in upper and lower grips of the tensile testing machine, their positioning by a screw (4) and fixing by the spindle (3). The samples of wire mesh are pining up on hooks (7), which are specially adapted high strength bolts (9) with appropriate nuts (8), positioned in the holes (6) on the basic plate (1) of the tool for ensuring safety testing by preventing slipping of samples during loading.

The complete set of this universal tool is made of two identical parts shown in Fig 1. Each part is made from basic steel plate, with $10 \mathrm{~mm}$ thickness, and with drilled holes. The cylindrical holes (6) are drilled in number defined in standard BS EN 10223$3: 2013$. The holders (5) are positioned on the plate (1) in a manner that is indivisible. The number of so-called „hooks“ (7) is defined by standard BS EN 10223-3:2013, too. The „hooks“ actually represents the specially adapted high strength bolts (9) with appropriate nuts (8) as shown in Fig 2. Each part of this universal tool is positioned in lower and upper grips of the tensile testing machine by gripping of the spindle (3). The dimensioning of all elements of this universal tool is calculated according to the diameter of wires and dimension of wire meshes. 


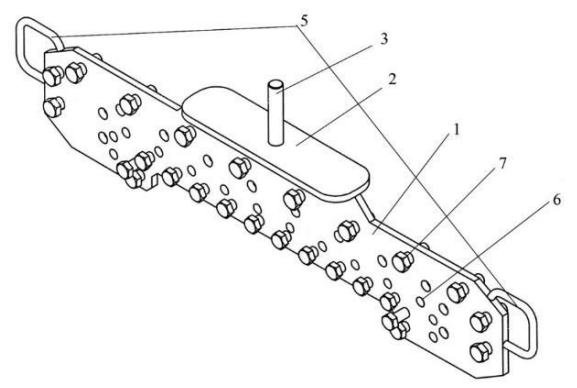

Fig. 1. Construction of developed universal tool for testing of tensile properties of hexagonal steel wire mesh.

Fig 2. shows in detail position of cylindrical holes on the plate (1) for testing standard dimensions of zinc plated wire mesh of following dimensions: $10 \times 12 \mathrm{~cm}$, $8 \times 10 \mathrm{~cm}, 6 \times 8 \mathrm{~cm}$ and $5 \times 7 \mathrm{~cm}$. A number of holes for each particular dimension corresponds to test requirement:

- For testing of samples with 8 fields (one field is one hexagon on mesh) for dimensions $10 \times 12 \mathrm{~cm}$ and $8 \times 10 \mathrm{~cm}$ is achieved by drilling 12 holes $(10 ; 11)$ on the plate (1) with additional so-called ,end hooks" with two holes (14); and

- For testing of samples with of 10 fields (one field is one hexagon on mesh) for dimensions $6 \times 8 \mathrm{~cm}$ and $5 \times 7 \mathrm{~cm}$ is achieved by drilling 14 holes $(12 ; 13)$ on the plate (1) with additional so-called „end hooks“" with two holes (15).

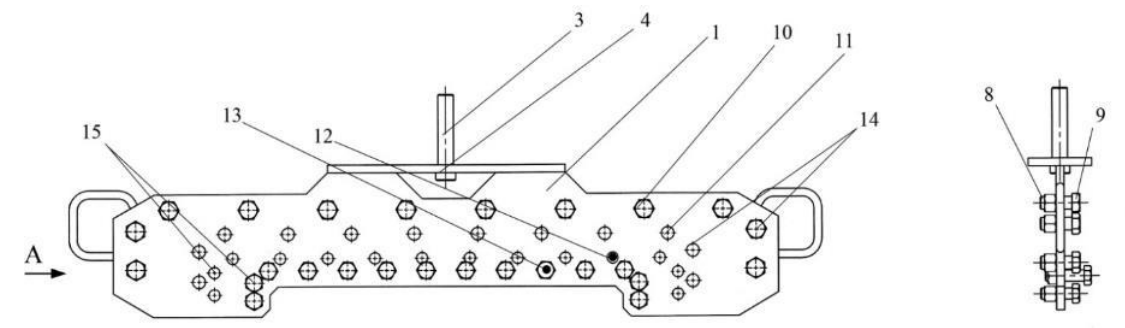

Fig. 2. The layout of the holes for hooks at the universal tool for testing of tensile properties of hexagonal steel wire mesh.

The production of "hooks" is based on standard high strength bolts, Fig 3, and it is the same for all "hooks". The novelty is the production of radius 16, shown in Fig 3. This radius is applied to "end hooks", too. Dimensioning of "hooks" is calculated in order to ensure safety requirements during testing up to force required for wire mesh rupture. Radius (16) decrease $20 \%$ load carrying capacity of standard high strength bolts and at the same time prevent sliding and dropping off the wire mesh during testing.

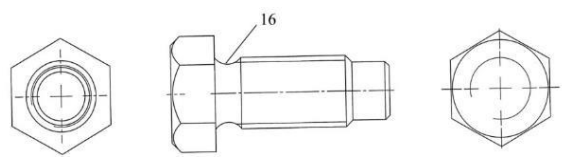

Fig. 3. Specially adapted high strength bolts. 
This universal tool is suitable because the same tool is quickly adjusted for testing of each specific dimension of wire mesh by changing space between upper and lower grips and quick change of "hooks". The Fig. 4 shows a schematic representation of starting position of hexagonal wire mesh on universal tool under unloaded condition. After positioning the sample part of wire mesh into appropriate "hooks" radius (Fig 3) the test setup is ready for increasing force and start of testing.

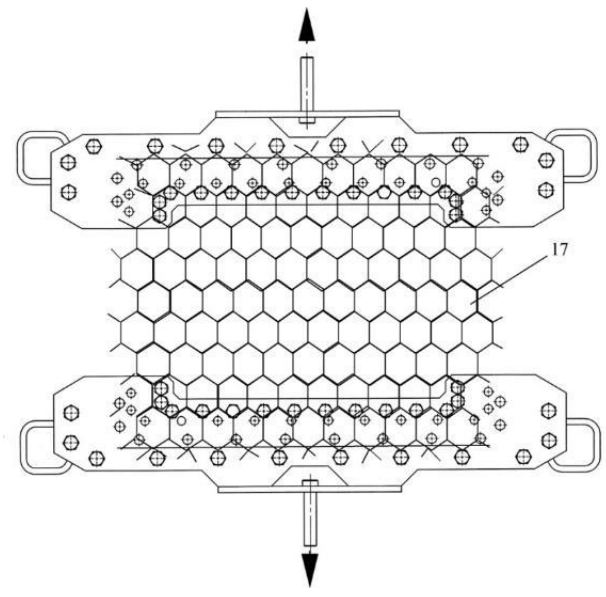

Fig. 4. Schematic drawing of one of the cases (wire mesh dimensions) and position of wire mesh on the universal tool.

\section{Finite Element Analysis of the developed tool}

The Finite Element Method is an appropriate numerical mathematical method for verification of the tool construction and calculation of the safety factor. In accordance with the vertical axis symmetry of the developed universal tool, the Finite Element Analysis is performed with a model of the half of tool. The modeled basic plate is shown in Fig. 5a. Fig. 5b shows the finite element mesh of the basic plate with finer mesh at the zones with stress concentration behavior (zones around the holes). The material characteristics used for modeling the analyzed tool following main characteristics are used: Young module of E=205GPa and Poisson's ratio of 0.3. The 3dimensional solid finite element type with midside nodes is used for modeling the main parts of the developed universal tool. The contact between hooks and holes on the basic plates are simulated with contact finite elements with midside nodes. The contact regions with stress concentration behavior are analyzed by non-linear solvers. The basic plate model has 31017 nodes and 5268 elements, while the whole model of the tool has 68991 nodes and 15988 elements. The volume models developed for different wire mesh dimensions with finite element models and boundary conditions definition are shown in Fig. 6 and 7. 


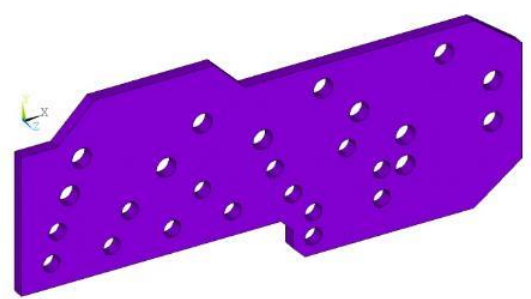

a)

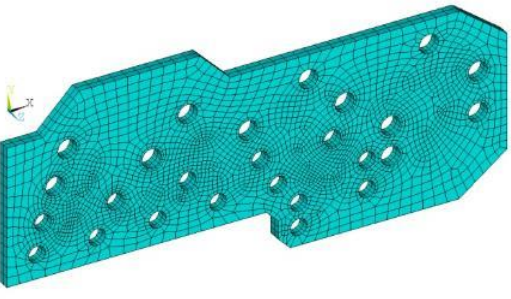

b)

Fig. 5. Volume and Finite element model of the tool basic plate.

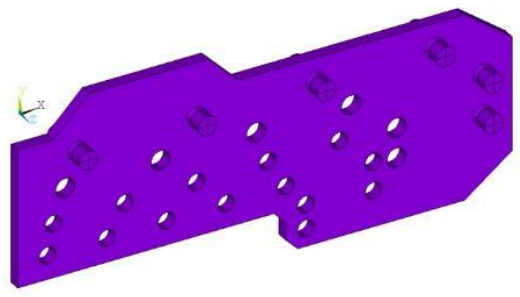

a)

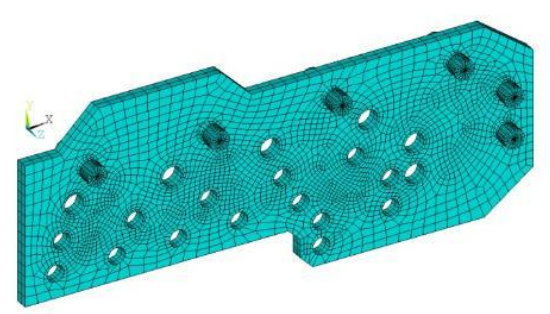

b)

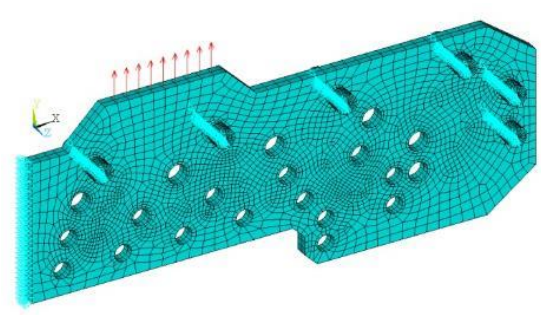

c)

Wire mesh type $10 \times 12$

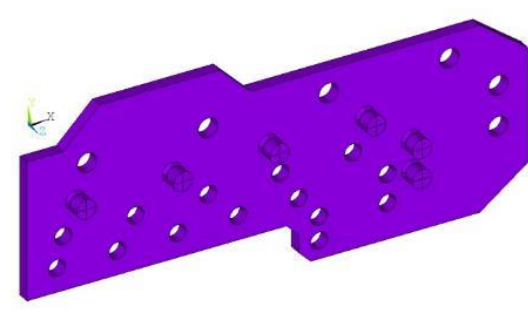

a)

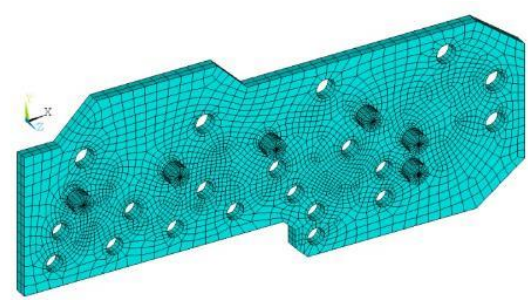

b)

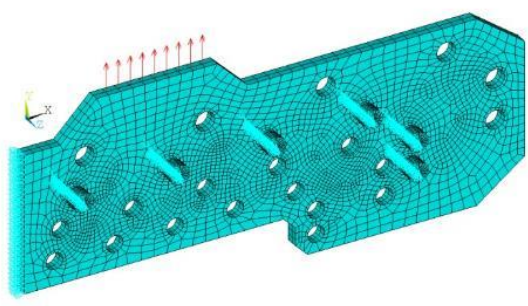

c)

Wire mesh type $8 \times 10$

Fig. 6. The volume models (a), finite elements models $(b)$ and boundary conditions $(c)$ developed for different wire mesh dimensions. 


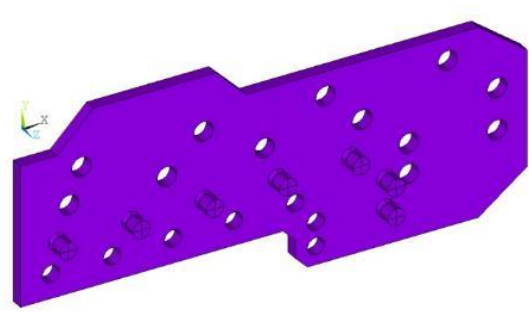

a)

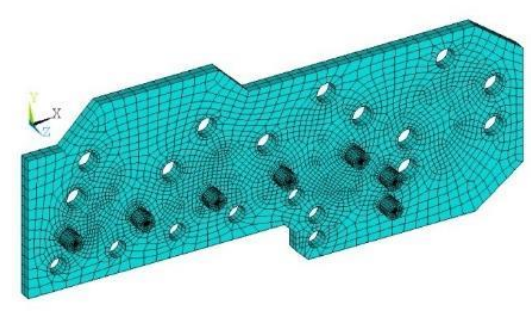

b)

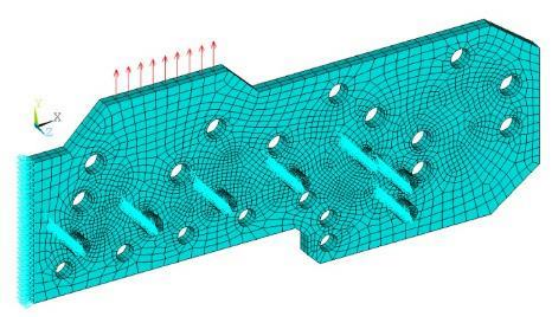

c)

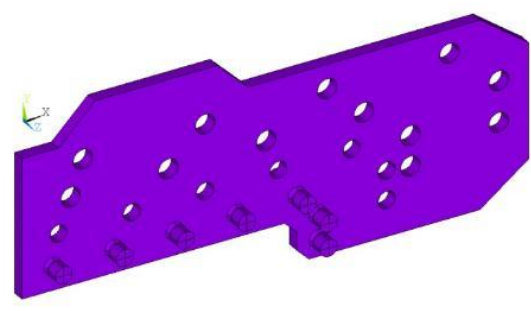

a)

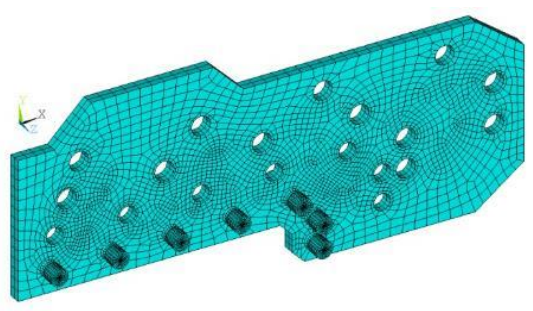

b)

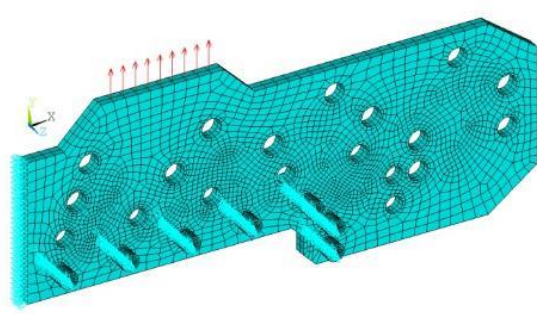

c)

Wire mesh type $6 x 8$

Wire mesh type $5 \times 7$

Fig. 7. The volume models (a), finite elements models (b) and boundary conditions (c) developed for different wire mesh dimensions.

The external load for tool verification is calculated on the base of the material characteristics and dimensions of the testing wire meshes. The considered tensile strength characteristics of the wires which are twisted in the mesh are multiplied by the number of mesh parts that are involved in the testing. The obtained stress is multiplied with the wires' cross-section and with the safety factor of 1,6. By this procedure, the value of $90 \mathrm{KN}$ is used as an external tensile load for FEA of the developed tool. 

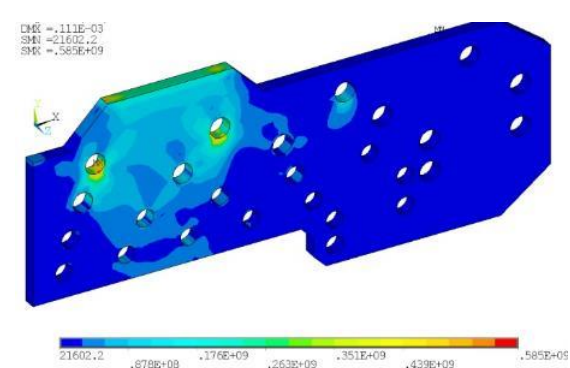

a)
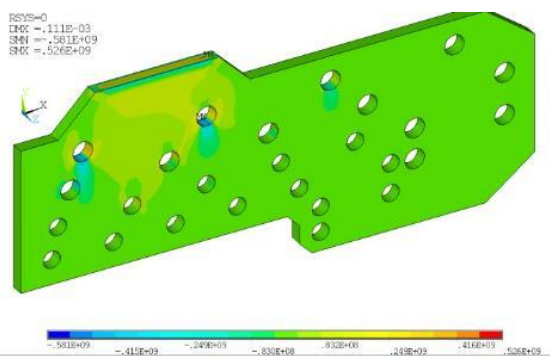

b)

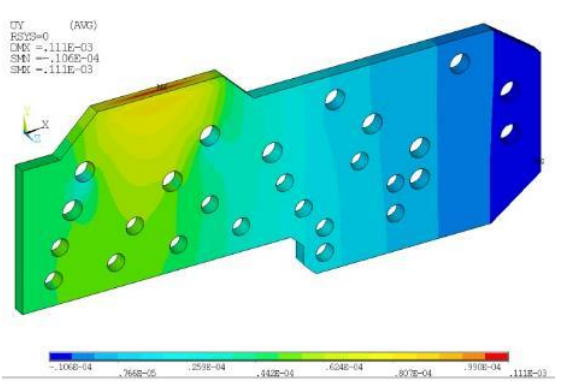

c)

Wire mesh type $10 \times 12$

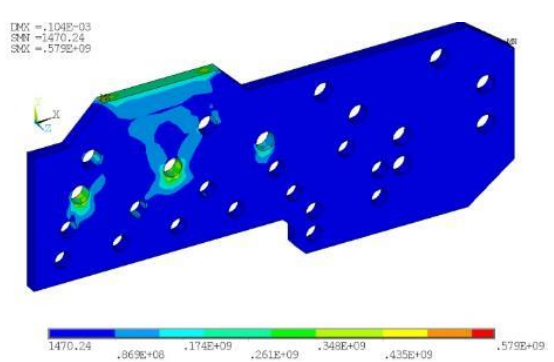

a)

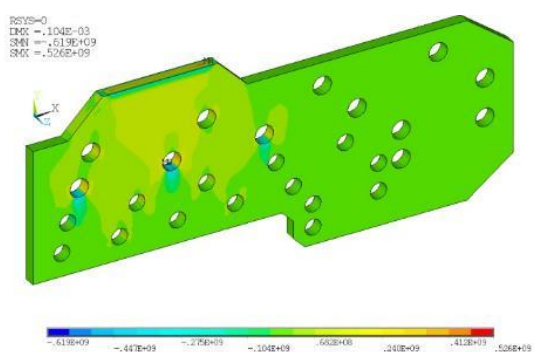

b)

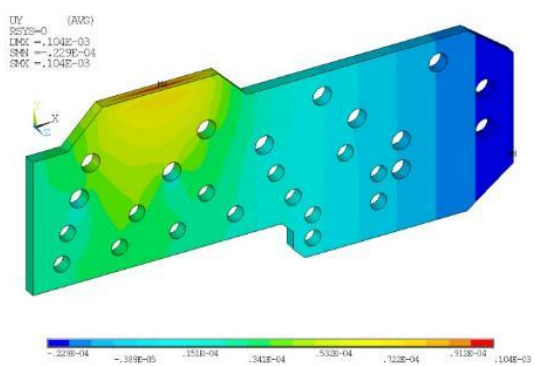

c)

Wire mesh type $8 \times 10$

Fig. 8. Results of the Finite Element Analysis: Equivalent VonMises stresses (a), tensile stresses $(b)$ tensile deformation $(c)$. 


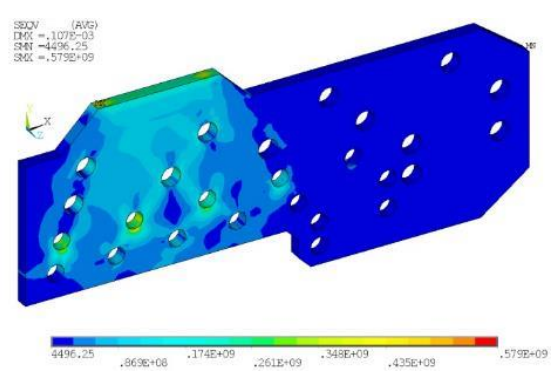

a)

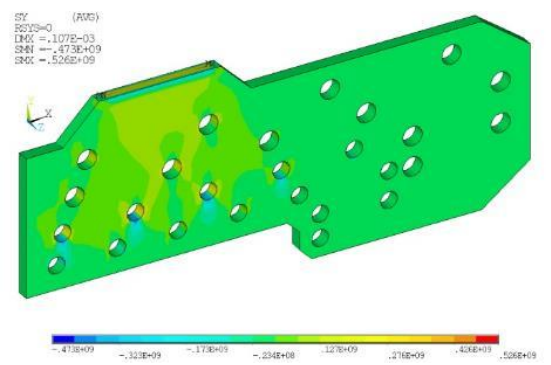

b)

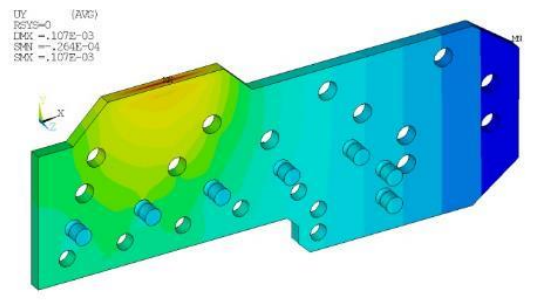

c)

Wire mesh type $6 \times 8$

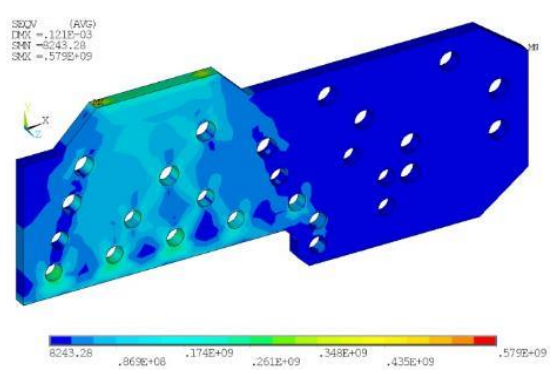

a)

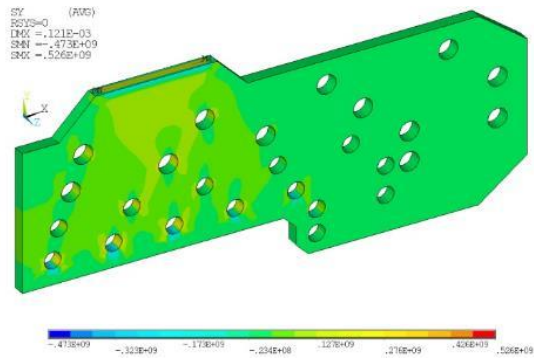

b)

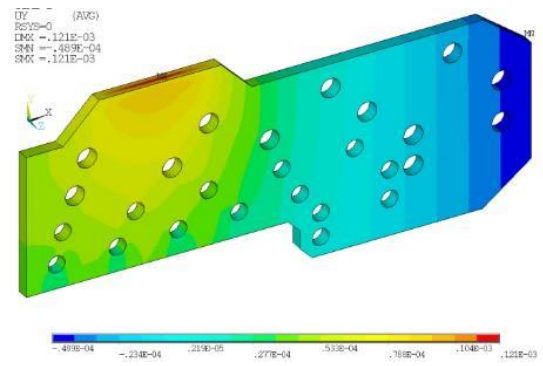

c)

Wire mesh type $5 \times 7$

Fig. 9. Results of the Finite Element Analysis: Equivalent VonMises stresses (a), tensile stresses (b) tensile deformation (c).

The maximal values for VonMises and tensile stresses obtained with Finite Element Analysis of the developed tool in the case of the pretension condition (loading with an external tensile load of $90 \mathrm{KN}$ calculated with a safety factor of 1,6) are given in Table 1 and represent the load capacity verification of the developed tool. 
I. Atanasovska et al. - Development of the Universal Tool for Testing of Tensile Properties ... 121

Table 1. Maximum stresses obtained by Finite Element Analysis.

\begin{tabular}{ccc}
\hline Wire mesh type & VonMises & Stress tensile-y \\
\hline $10 \times 12$ & $585 \mathrm{MPa}$ & $581 \mathrm{MPa}$ \\
$8 \times 10$ & $497 \mathrm{MPa}$ & $619 \mathrm{MPa}$ \\
$6 \times 8$ & $369 \mathrm{MPa}$ & $422 \mathrm{MPa}$ \\
$5 \times 7$ & $312 \mathrm{MPa}$ & $343 \mathrm{MPa}$ \\
\hline
\end{tabular}

\section{Conclusions}

This paper describes the design of a universal tool for testing of tensile properties of hexagonal steel wire mesh, which meets appropriate standard requirements and allows testing on all tensile testing machines with suitable test range. The fundamental design point of this universal tool is a high level of availability to obtain a high level of quality control in production or on construction site. With this new universal tool, it is possible to test series of different hexagonal steel wire meshes of different dimensions with a fast setup which is present very cost-effective approach compared with existing tools. The other advantages of this universal tool are:

- lower cost of construction of universal tool made of plate simply machined;

- reduced weight and ergonomic handling before and after finishing testing of wired mesh;

- fast setup for different testing requirements;

- simple positioning of mesh on the universal tool by positioning on modified bolts;

- can be easily modified after testing for use on different dimensions of wired mash;

- reduced personnel number for the test;

- increased safety during testing due to minimized sliding and dropping of wired mesh during testing;

- increased reliability of test results and reduced data scatter due to the high level of repeatability of test conditions.

Application of this universal tool is significant due to increased requirements for passive safety on roads and preventing falling rocks and stones from slopes. This universal tool reduces time and costs of testing of large series of wire meshes as a quality control measure for positioning steel wire meshes and slopes, particularly in mountain regions.

\section{References}

[1] Standard BS EN 10223-3:2013: Steel wire and wire products for fencing and netting. Part 3: Hexagonal steel wire mesh products for civil engineering purposes

[2] N. Sasiharan, B. Muhunthan, T. C. Badger, S. Shu, D. M. Carradine: Eng Geo, 88 (2006) 121-132.

[3] Morton E.C., Thompson A.G. and Villaescusa E., In: Proceedings of ISRM Congress, 2007.

[4] D. Bertrand, F. Nicot, P. Gotteland, S. Lambert: Canadian Geotechnical Journal, 45 (2008) 1104-1117. 
[5] Angeli s.r.l., Wire-test, http://www.angelisrl.eu/en/product/special/mechtest.shtml, 14.05.2018.

[6] Jinan Heng Rui Testing Machine Co, Gripper for Machine Woven Wire Mesh Used in Construction, http://www.jnshiyanji.com/en/Pro show.asp?Proid=480, 14.05.2018.

[7] Atanasovska I. et al., The universal tool for testing characteristics of hexagonal steel wire mesh for civil engineering purposes. R.S. Patent Application number MP-2018/0012, filed April 4, 2018.

\section{(c) (i) Creative Commons License}

This work is licensed under a Creative Commons Attribution 4.0 International License. 